\title{
Germanica
}

\section{Literaturkritik als Denkraum. Ijoma Mangold im Gespräch mit Stephanie Baumann und Bénédicte Terrisse}

Stephanie Baumann et Bénédicte Terrise

\section{(2) OpenEdition}

Journals

Édition électronique

URL : https://journals.openedition.org/germanica/8437

DOI : 10.4000/germanica.8437

ISSN : 2107-0784

Éditeur

Université de Lille

Édition imprimée

Date de publication : 1 décembre 2019

Pagination : 147-160

ISBN : 978-2-913857-44-5

ISSN : 0984-2632

\section{Référence électronique}

Stephanie Baumann und Bénédicte Terrise, „Literaturkritik als Denkraum. ljoma Mangold im Gespräch mit Stephanie Baumann und Bénédicte Terrisse", Germanica [Online], 65 | 2019, Online erschienen am: 01 Januar 2022, abgerufen am 08 Januar 2022. URL: http://journals.openedition.org/germanica/8437 ; DOI: https://doi.org/10.4000/germanica.8437

(c) Tous droits réservés 


\section{Entretien}




\section{Literaturkritik als Denkraum. Ijoma Mangold im Gespräch mit Stephanie Baumann und Benedicte Terrisse}

Ijoma Mangold ist ein deutscher Literaturkritiker der Wochenzeitung Die Zeit. Er studierte Literaturwissenschaft und Philosophie in München und Bologna. Seit 2001 arbeitete er als Feuilletonredakteur bei der Süddeutschen Zeitung und seit 2009 als stellvertretender Leiter des Feuilletons der Zeit. Seit 2018 ist er deren kulturpolitischer Korrespondent in Berlin. Er moderierte mit Amelie Fried die Literatursendung Die Vorleser im ZDF und seit 2013 gehört er zum lesenswert-Quartett im SWR. Im Jahr 2008-2009 war er Gastprofessor für Literaturkritik an der Georg-August Universität Göttingen, und 2014-2015 Gastprofessor an der Universität Saint Louis, USA.

Stephanie Baumann: Herr Mangold, Sie haben eine Gemeinsamkeit mit Marcel Reich-Ranicki: beide haben Sie autobiographische Bücher geschrieben. In Das Deutsche Krokodil (2017) streifen Sie Ihre Arbeit als Literaturkritiker jedoch nur am Rande. Wie sind Sie Literaturkritiker geworden? Und hat Marcel Reich-Ranicki dabei auf irgendeine Weise eine Rolle gespielt?

Ijoma Mangold: Damals war er für mich vermutlich eher ein GegenModell. Während des Studiums hatte das Feuilleton eine starke Anziehungskraft auf mich ausgeübt und das hing mit dem Feuilleton der $F A Z$ zusammen. Das las ich und fand ich ungeheuer inspirierend, weil hier die Gegenwart gleichzeitig mit akademischem Ernst und mit dem Spielerischen des Feuilletons beschrieben wurde. Ich glaube, in den neunziger Jahren war eine besonders originelle Generation von

GERMANICA, 2019, LXV, pp. 1 à 16. 
Journalisten bei der FAZ. Diejenigen, die in den letzten Jahren der Herausgeberschaft von Joachim Fest das Feuilleton prägten, waren der Literaturchef Frank Schirrmacher, der später Herausgeber wurde, und der Sachbuchchef Gustav Seibt. Wenn man ehrlich ist, haben sie eigentlich einen Anti-Ranicki-Kurs gefahren. In späteren Jahren hat Schirrmacher dann mit Reich-Ranicki wieder eine Allianz geschlossen. Aber in den 1990er Jahren sagte man eher, der Literaturbegriff von Marcel ReichRanicki ist uns - so lautete damals das Schimpfwort - zu sehr „sozialistischer Realismus“. Reich-Ranicki war berühmt dafür, dass er starke Intellektualisierungen der Literatur nicht wertschätzte. Sein beliebtestes Beispiel war Robert Musils Mann ohne Eigenschaften, dort wo die Literatur in eine Art Essayismus übergeht. Das hat er nicht als literarische Stärke, sondern als Schwäche angesehen. Dann war er damals natürlich auch schon der Mann des Fernsehens. Seinerzeit gab es noch eine stärkere kulturelle Polarität zwischen dem alten Bürgertum, das sola scriptura - auf Papier baute, und der Fernsehwelt. Das gehörte nicht so selbstverständlich zusammen wie heute. Reich-Ranicki hat bis 1988 den Literaturteil geleitet, und danach ging er in Rente. Der Nachfolger wurde Frank Schirrmacher, und das war eine ganz andere Form der Literaturkritik. Sie zielte nicht so sehr auf die rhetorischen Effekte ab, sondern war mit einem kulturhistorischen Interesse und Hintergrund versehen. Jetzt habe ich sehr weit ausgeholt. Aber um zu erklären, warum ich Literaturkritiker wurde, würde ich sagen, dass die Bedeutung, welche die tägliche Lektüre des FAZ-Feuilletons für mich als Student hatte, sehr entscheidend war. Und dieses FAZ-Feuilleton war gerade kein ReichRanicki-Feuilleton, sondern das post-Reich-Ranicki-Feuilleton.

S.B.: War Ihr primärer Zugang zum Feuilleton die Literaturkritik? Es umfasst ja ganz unterschiedliche Formen und Gegenstände.

I.M.: Das stimmt. Aber das habe ich nicht so stark getrennt. Ich fand schon immer, dass der Literaturkritiker gleichzeitig jemand ist, der zur Gegenwartsanalyse fähig sein sollte. Und es ist auch reizlos, über eine Neuerscheinung zu schreiben, wenn man sie nur literarisch beschreibt, ohne sie als Symptom der Epoche, der Zeit, der Gegenwart, in der man lebt, zu deuten. Insofern stand das Feuilleton der FAZ damals für das, was man das ,politische Feuilleton“ nannte. Alle waren sehr leidenschaftlich mit dem Gegenstand ihrer Künste befasst, haben aber immer die Brücke zur Gegenwartsanalyse geschlagen.

S.B.: Ein Feuilleton, das kulturelle Gegenstände als Zeitsymptome deutet: das erinnert an das Feuilleton, wie es Siegfried Kracauer in den 1920er Jahren auffasste. 
I.M.: Absolut. Man kann sagen, das war ein Kracauer-Feuilleton. War Kracauer nicht früher auch bei der Frankfurter Zeitung? Nachdem ReichRanicki in Rente gegangen war, hatte er noch ein Zimmer und betreute die Frankfurter Anthologie. Aber die Nachfolger wollten ein anderes literaturkritisches Paradigma durchsetzen. Wenn eine neue Generation anrückt, will sie alles anders machen als der unmittelbare Vorgänger. Und man konnte sich von Reich-Ranicki auch gut abgrenzen, indem man sagte: wir machen Zeitung, und er macht jetzt Fernsehen.

S.B.: Wie Sie einmal in einem Interview betont haben, kann man in Ihrem Fall, anders als bei Reich-Ranicki, nicht von einem bikulturellen Hintergrund sprechen. Sie haben dort auch erzählt, dass Ihnen beim Verfassen Ihres Buches die lebensgeschichtlichen Hintergründe gewisser habitueller Eigenheiten bewusst geworden seien, darunter Ihr sprachlicher Habitus. Gibt es einen Zusammenhang zwischen dieser Sprachsensibilität, die Sie mit der Ihnen zugeschriebenen bikulturellen Identität in Zusammenhang bringen, und Ihrem Interesse für Literatur? Anders gefragt: erzählt Ihr Buch implizit auch die Geschichte einer „Berufung"?

I.M.: Im Deutschen Krokodil habe ich meine Geschichte auch in diesem Sinne erzählt: Wenn man nicht aussieht wie ein Deutscher, ist der einzige Weg sicher zu stellen, dass über den Pass keine Zweifel entstehen, dass man in einem sehr gestochenen Hochdeutsch einen syntaktisch anspruchsvollen Satz sagt. Dann ist klar, dass man zur Gesellschaft gehört, es ist sozusagen die Zugangsberechtigung. Insofern findet sich im Deutschen Krokodil in der Tat die Erzählung, dass ich Sprache immer auch als Instrument der Anerkennung genutzt habe, um gewissermaßen in die richtige Schublade eingeordnet zu werden. Dass sich aus diesem Sprachbewusstsein vielleicht auch eine Nähe oder Begeisterung zur Literatur ergab, würde ich in dieser Kausalität jedoch nicht sagen wollen. Es kann auch sein, dass ich Freude oder Begeisterung an der Literatur hatte, dass sich dadurch mein Sprachbewusstsein ausbildete und mir plötzlich aufging, ah - die Sprache lässt sich auch als Faktor der gesellschaftlichen Anerkennung nutzen. Was das Ei und was die Henne ist, ist wie bei den meisten komplexen Vorgängen, die mit einer unberechenbaren Dynamik arbeiten, nicht zu sagen.

Mich hat aber schon als Jugendlicher immer fasziniert, über das deutsche kulturelle Gedächtnis - im Sinne Aleida Assmanns - besser Bescheid zu wissen als meine Mitschüler. Dies gab mir das Gefühl, ich kann mitentscheiden, was Deutschland ist, in dieser historischen Perspektive. Das mag tatsächlich eine Parallele mit Reich-Ranicki sein. Er, der im Warschauer Ghetto den Nationalsozialismus überlebt, von den Deutschen, die es auf sein Leben abgesehen hatten, nicht als Deutscher 
anerkannt wird, er weiß, dass in Wahrheit das eigentliche kulturelle Erbe Deutschlands bei ihm, nämlich in seinem Kopf, in seinem umfassenden Lektürekanon am besten aufgehoben und konserviert ist. Insofern gibt es eine ähnliche Spannung. Nur habe ich in Deutschland nie eine Form der Ausgrenzung erlebt. Deswegen ist bei mir, ganz anders als bei ReichRanicki, alles vollständig tragikfrei. Aber dieses Moment, dass der, der nicht zu Deutschland gehören soll, eigentlich Träger des kulturellen Erbes ist, ist eine interessante biographische Figur bei Reich-Ranicki und in der freundlichen Version kann man diese auch in mein Krokodil hineinlesen.

S.B.: Reich-Ranicki erwähnt in einem Essay über Literaturkritik mit dem Titel "Nicht nur in eigener Sache" in Lauter Verrisse Goethes Unterscheidung einer zerstörenden und produktiven Art von Kritik. Erstere messe das Werk an einem abstrakten Maßstab, um darzulegen, warum es nichts tauge. Letztere messe das Werk an dem Maßstab, den sich der Autor vorgesetzt habe, um diesen auf Momente des Scheiterns hinzuweisen, damit er sich verbessern könne. Schreiben Sie auch manchmal für Autoren?

I.M.: Natürlich denke ich in erster Linie an den Leser. Eine Kritik ist ein Text, den man selber schreibt und er muss funktionieren. Ein Text funktioniert, wenn er seinen Leser mitzieht. Das ist nicht der Verfasser des Romans, über den man schreibt. Gleichwohl im Fall eines Lobes - im Fall eines Verrisses ist es ganz anders, - würde es mich glücklich machen zu wissen, dass die Art, wie ich ein Buch beschrieben habe, vom Autor geschätzt wird. Dass er sieht, dass hier etwas gesehen worden ist, was ihm auch vorschwebte. Dass vielleicht sogar etwas gesehen wurde, was ihm nicht vorschwebte, denn jedes Buch ist immer reicher als die Intention seines Autors.

\section{S.B.: Also produktive Kritik im Sinne eines Gesprächs über das Werk?}

I.M.: Alles Pädagogische liegt mir völlig fern. Ich glaube, ich bin ein sehr diskursiver und überhaupt kein pädagogischer Mensch. Ich habe immer Lust, in jede Auseinandersetzung, in jeden Austausch einzusteigen. Aber ich habe nie den Hintergedanken ,Jetzt habe ich dem Autor aber eine kluge Lektion gegeben. Hoffentlich beherzigt er es, damit sein nächster Roman noch besser wird“. Marcel Reich-Ranicki hatte manchmal tatsächlich diese Vorstellung - vor allem mit Blick auf Martin Walser, dessen Romane ihm immer zu lang waren -, er hätte Ratschläge parat, und wenn Herr Walser sie beherzigen würde, wäre das nächste Werk besser. Und als er dann über Das fliehende Pferd jubelte, eine schmale Novelle, war er tatsächlich überzeugt, dass dies zum guten Teil auf ihn und seine Kritik zurückzuführen sei. Er meinte, jetzt habe Walser endlich einmal beachtet, was er in seinen Rezensionen schon immer bemängelt hatte. Das ist für 
mich eine völlig ferne Welt. Ich habe vielleicht einen pädagogischen Hang in dem Sinne, dass man sich gegenseitig für einen Gegenstand und für die Auseinandersetzung darüber begeistert. Aber dass man mitgibt, was jemand anderes umsetzen soll... Ehrlich gesagt schreibe ich eine Rezension nicht einmal, weil ich möchte, dass möglichst viele Leute das Buch lesen. Ich möchte, dass möglichst viele Leute die Rezension lesen, um sich mit der Rezension auseinanderzusetzen.

\section{S.B.: Aber doch auch im Sinne einer Orientierungshilfe?}

I.M.: Ja, das ist zwingende Pflichtaufgabe der Literaturkritik, die wir erfüllen müssen. Gleichwohl ist mein Hauptimpuls bei einer Rezension, einen diskursiven Raum zu eröffnen, in dem der Leser am Beispiel eines Romans zum Nachdenken über die Welt gebracht wird.

S.B.: Im Gespräch mit Joachim Fest hat Marcel Reich-Ranicki seine Urteilsweise einmal auffolgende Formel gebracht: „Ein Buch, das mich interessiert, halte ich für gut, eines, das mich langweilt, für schlecht. Ich suche dann rationale Argumente, die meine Reaktion auf dieses Buch objektivieren und begründen "1. Wie stehen Sie zu dieser Aussage?

I.M.: Diesbezüglich habe ich eine Entwicklung durchgemacht. Als ich anfing, Literaturkritiker zu sein, rümpfte ich über Reich-Ranickis grob rhetorischen Stil ein wenig die Nase, weil es mir oft an ästhetischen Differenzierungen fehlte. Heute würde ich sagen, dass mein Respekt vor seiner rhetorischen Zuspitzung mit den Jahren immer mehr gewachsen ist und dass ich heute vielleicht auch etwas mehr dazu neige, auf die Sahne zu hauen, als ich es vielleicht vor zwanzig Jahren getan hätte. Natürlich auch deswegen, weil ich selber nun ja auch schon länger im Fernsehen Literaturkritik mache. Da hat Reich-Ranicki wirklich den Maßstab gesetzt und ich würde jederzeit sagen, dass man sich hier an ihm orientieren muss. Das würde ich über seine schriftlichen literaturkritischen Rezensionen nicht sagen. Das könnte bei anderen funktionieren, aber das ist nicht mein Stilideal. Beim Fernsehen hingegen ist es state of the art. Hier muss man größer auftreten, und dafür hatte Reich-Ranicki früh einen Sinn. Als der damalige Herausgeber Joachim Fest 1973 ReichRanicki zur FAZ holte, um den damaligen Literaturchef Karl Heinz Bohrer zu ersetzen, war die Idee, das Feuilleton und die Literaturkritik in der FAZ populärer zu machen. Karl Heinz Bohrer ist jedoch in gewisser Weise mein role model. Er war eher so der Typus ,intellektueller Snob", wofür ich ihn sehr bewundere und mag. Er hatte kein Problem damit Rezensionen abzudrucken, die am Rande der Verständlichkeit

1. _ Zwischen Diktatur und Literatur. Marcel Reich-Ranicki im Gespräch mit Joachim Fest, Frankfurt a.M., Fischer Taschenbuch Verlag, 1994, S. 100. 
waren, weil sie stark von Literaturtheorie beeinflusst waren. Das war der Paradigmenwechsel mit Reich-Ranicki: so wie er von Literatur verlangte, dass sie nicht langweile, verlangte er von der Literaturkritik, dass sie leicht verständlich, amüsant oder packend sein solle.

S.B.: Joachim Kaiser hat einmal in einem Gespräch mit Reich-Ranicki gesagt, bei diesem werde stets 10 zu 1 gewonnen oder verloren. Während es ihm selbst um Wahrheit gehe, sei es Reich-Ranicki vornehmlich um Wirkung zu tun. Wirkung oder Wahrheit? Wo würden Sie sich selbst einordnen?

I.M.: Das ist gut gesehen. Die Beschreibung von Joachim Kaiser ist absolut zutreffend. Ich hatte in meiner Zeit bei der Süddeutschen Zeitung auch das Vergnügen, Kaisers Kollege sein zu dürfen. Er war im Ruhestand, aber er war immer noch da und nahm an unseren Sitzungen teil. Er war ein pointierter Feuilletonist, aber seine Differenzierungsgabe war größer als die von Marcel Reich-Ranicki. An der Dichotomie, die er hier aufmacht, Wirkung oder Wahrheit, ist schon etwas dran. Ich weiß gar nicht, wo ich mich einordnen soll. Mittlerweile würde ich sagen, dass die Wirkungsversessenheit mich auch manchmal im Griff hat, namentlich im Fernsehen, weil Sie im Fernsehen ein $5 \mathrm{zu} 4$ rhetorisch nicht über die Bühne bekommen. Das funktioniert nicht. Selbst wenn Sie es versuchen, wird das Publikum der Meinung sein, Sie haben den Daumen nach oben gehoben oder eben nach unten gesenkt. Die Wirklichkeit ist grau. Aber das lässt sich nicht vermitteln. Ein $10 \mathrm{zu} 1 \mathrm{gibt}$ es sehr selten in der Literatur. Natürlich gibt es völlig missratene Romane, aber dann ist ja die Frage, warum man überhaupt über sie schreibt. Es soll deswegen nicht langweilig werden, aber ein ,ja aber"leuchtet mir theoretisch mehr ein. $\mathrm{Ob}$ ich mich praktisch daran halte, weiß ich nicht.

S.B.: Sie haben zu einem Band mit Texten von Fritz Raddatz mit dem Titel Stahlstiche ein Vorwort geschrieben. Dort heißt es, der Verriss sei , eine Regierungserklärung, ein Machtanspruch, ein Fehdehandschuh, eine Selbstoffenbarung und eine Selbstinszenierung zugleich"2. Mir schien es, als würden Sie sich von der Form des Verrisses ein wenig distanzieren.

I.M.: Nein, gar nicht. Der Verriss ist die Königsdisziplin der Theaterkritik. Die Theaterkritik ist noch schärfer in der Überpointierung des Verrisses als die Literaturkritik. Das gehört zum Genre und ist historisch seit Alfred Kerr so gewachsen. Noch mehr Blut fließt in der Theaterkritik, fast keines in der Kunstkritik. Warum, weiß ich nicht. Es kann keinen Feuilletonjournalismus ohne Eitelkeit und Spielfreude geben. Und die

2. — Ijoma Mangold, „Vorwort“, in: Fritz J. Raddatz, Stahlstiche. 35 Einreden aus 35 Jahren, Hamburg, Rowohlt Verlag, 2013, S. 7-16, hier S. 7. 
kann sich im Verriss ideal austoben. Allerdings funktioniert der auch nur, wenn er einen großen Gegenstand hat. Aus dem mittelmäßigen Debüt können Sie auch kein Verrissfeuerwerk entfachen, aus dem neuen Günter Grass - um in der Epoche von Reich-Ranicki zu bleiben - hingegen durchaus. Der Verriss von mir, der am stärksten öffentlich wahrgenommen worden ist, wurde nicht wegen seiner eigenen Qualitäten wahrgenommen, sondern weil der Gegenstand ein neuer Roman von Günter Grass war, dieses Zwiebel-Buch. Man braucht die Fallhöhe, damit die Leute sagen: „Wow! Das ist er aber hart angegangen.“

S.B.: Fließt in der Theaterkritik womöglich mehr Blut als in der Kunstkritik, weil das Theater traditionsgemäß die politischere Kunstform ist? Und hat nicht der Verriss in der Literaturkritik auch oft mit Politik zu tun?

I.M.: Das kann sehr gut sein. Natürlich nicht zugegebenermaßen. Zugegebenermaßen würde jeder behaupten, dass er aufgrund von ästhetischen Kriterien urteilt, aber selbstverständlich ist das sehr oft politisch grundiert. Reich-Ranickis Verriss von Günter Grass' Ein weites Feld ist aus meiner heutigen Sicht ästhetisch völlig berechtigt gewesen. Aber die Schärfe des Verrisses hatte mit Grass' Ablehnung der deutschen Wiedervereinigung zu tun.

S.B.: Sie sprachen vorhin von einem politischen Feuilleton nach ReichRanicki. Hat Reich-Ranicki auf seine Art nicht auch eine politische Rolle gespielt?

I.M.: Nein. Weder bin ich der Meinung, dass er eine politische Rolle gespielt hat, noch meint der Begriff politisches Feuilleton, dass man sich mit einer engagierten Position politisch einsetzt. Fast eher im Gegenteil. Das traditionelle Feuilleton besteht aus den Genres Literaturkritik, Theaterkritik, Kunstkritik. Und Joachim Fest fing an, zu sagen: unser feuilletonistischer Blick auf die Gegenstände der Politik ist ein anderer als der Blick der politischen Redakteure. Dann begannen plötzlich auch Feuilleton-Redakteure über jene Gegenstände zu schreiben, die weiter vorne die politischen Redakteure behandelten. Aber auf eine andere Art und Weise, ohne deswegen mit einer ideologischen Duftmarke versehen zu sein. Das Musterbeispiel eines politischen Feuilletons wäre z.B. Karl Heinz Bohrers Kolumne über Provinzialismus. Er hat eine Physiognomie von Helmut Kohl entworfen. Das war politisches Feuilleton, weil ein Feuilletonist über eine politische Figur schrieb. Er tat das aber mit den Mitteln des Feuilletons, physiognomisch, indem er den Typus Helmut Kohl phänomenologisch zu beschreiben versuchte, was der politische Leitartikel gar nicht tun würde. Es war der große Historikerstreit von 1986/87, der das ,politische Feuilleton“ begründete. Dazu trug auch bei, dass unter den Feuilletonisten viele Historiker waren, die damals also 
versuchten, Gegenwartsereignisse historisch zu erklären. In diesem Sinne hat Marcel Reich-Ranicki nie politisches Feuilleton gemacht. Er hat einfach Rezensionen geschrieben. Ob er darin eine politische Figur war? Da müsste man vorerst klären, was eine politische Figur ist: jemand mit einer politischen Meinung oder jemand, der tatsächlich von außen betrachtet eine politische Rolle spielt. Wenn Reich-Ranicki von außen betrachtet eine politische Figur war, in diesem objektiven Sinne, dann sehr spät, nach seiner Autobiographie. Da hatte Deutschland in ihm das, was es vernichtet hatte, gewissermaßen als letztes Exemplar vor sich: die deutsch-jüdische Kultursymbiose, von der wir wissen, dass es sie nie gegeben hat. Aber das Ideal konnte man in ihm noch einmal sehen. Dass er privat irgendeine politische Meinung hatte, ist für sein Werk und Schaffen uninteressant. Nach der Gruppe 47 waren all diese Leute in einem bestimmten Sinne sozialdemokratisch. Das wird auch bei ReichRanicki der Fall gewesen sein, aber das hat ihn nicht interessiert. Es würde nichts Charakteristisches über ihn sagen, wenn man sagte, ,er war ein Linker". Da würde man nicht viel gewinnen. Natürlich war er kein Rechter. Aber war er ein Linker?

S.B.: Kommen in der Debatte über Ein weites Feld nicht die beiden von Ihnen genannten Aspekte zusammen: handelt es sich nicht um einen Ausläufer jener Selbstverständigungsdebatten, die in den 1990er Jahren und seit dem Historikerstreit über Identität, Nation, Wiedervereinigung etc. geführt wurden, die hier nun auch im Rahmen von Literaturkritik ausgetragen wurden?

I.M.: Da haben Sie völlig recht. Im Übrigen hat Reich-Ranicki in den 1990er Jahren nicht mehr sehr viel geschrieben. Seine Rezensionen dieser Jahre kann man an einer Hand abzählen, eine davon war die über Ein weites Feld. In der Tat war diese Debatte im Rahmen der deutschen - wie Sie sagen - „Selbstverständigung“ zentral. Er war aufgrund seiner eigenen Totalitarismuserfahrung, nicht nur mit Blick auf Nazi-Deutschland, sondern auch mit Blick auf das kommunistische Polen nach 1945, über Günter Grass’ Rede von der DDR als „kommoder Diktatur“ aus eigener Erfahrung empört. Das fand er sträflich naiv.

S.B.: Es wird stets betont, das Literarische Quartett habe einen nachweisbaren Einfluss auf die Verkaufszahlen der besprochenen Bücher gehabt. Hat die Sendung Ihrer Meinung nach auch zur Debattenkultur oder zur Schaffung einer literarischen Öffentlichkeit beigetragen? Und worin liegt der eigentliche Unterhaltungscharakter des Formats?

I.M.: Man kann das Literarische Quartett auch losgelöst von den Büchern betrachten und es als Schaukampf würdigen, wie die debating clubs in Oxford und Cambridge. Diese rhetorisch performative Qualität 
des Miteinanderstreitens hat die Sendung vorbildlich vorgeführt. Die Annahme, dass es die Deutschen diskursiver gemacht hätte, würde jedoch die Wirkung des Formats überschätzen. Die Öffentlichkeit, die ReichRanicki geschaffen hat, war nicht eine literarische Öffentlichkeit - die hat es schon immer gegeben - sondern eine zusätzliche Öffentlichkeit, eine Fernsehöffentlichkeit für die Literatur. Nicht in dem Sinne, dass diese in erster Linie am Buch interessiert war, sondern an der Figur des Literaturkritikers bzw. an der theatralischen Konstellation, dass sich vier Literaturkritiker streiten können. Es ist ihm gelungen, dass der etwas hermetische Beruf des Literaturkritikers bekannt wurde, und das hat uns Nachfolgern das Leben unendlich vereinfacht. Wenn man mich nach meinem Beruf fragt, kann ich davon ausgehen, dass die Leute wissen, was ich mache.

Literaturkritik im strengen Sinne hingegen ist noch nie Anlass einer Debatte gewesen. Es gibt Debatten, in deren Zentrum Schriftsteller stehen. Aber das sind keine Debatten über Literatur, sondern immer Debatten über die moralische Bewertung der Figur des Autors. Das beste Beispiel haben wir jetzt mit der Debatte über Peter Handke. Mit der Literarizität seiner Romane hat das nichts zu tun, was schon daran erkennbar ist, dass 90 Prozent derer, die sich - völlig zu Recht, das dürfen sie gerne -, an dieser Debatte beteiligen, noch nie auch nur einen Satz von Peter Handke gelesen haben. Wenn Literaturkritik etwas mit ästhetischer Form zu tun hat, dann ist sie nicht debattenfähig, weil über ästhetische Formen in Wahrheit nicht in einem breiten Sinne gestritten wird. Ich habe auch noch nie einen Leserbrief auf eine Buchrezension bekommen. Wenn ich hingegen meine Meinung über Gott und die Welt sage, kriege ich sie tausendfach, weil es auch viel leichter ist, einer Meinung über Gott und die Welt eine andere Meinung entgegenzusetzen. Eine literaturkritische Debatte wäre sehr anstrengend: Sie müssten erst einmal das Buch lesen, die Einstiegsschwelle zu einer literaturkritischen Debatte ist einfach sehr hoch. Ich habe es nun ein bisschen pointiert. Wenn ich an die Debatte über Karen Köhlers Roman Miroloi denke - sie fand vor vier Monaten statt - war das tatsächlich sehr nah an ästhetischen Fragen. Das ist dann immer sehr schön. Aber in der Regel - nehmen wir Martin Walsers Tod eines Kritikers oder Peter Handkes Nobelpreis - sind es Debatten über den moralischen Status des Schriftstellers, nicht aber über seine literarische Kraft.

S.B.: Ich glaube nicht, dass der Unterhaltungscharakter des Literarischen Quartetts lediglich darin liegt, dass es sich um eine Art Schaukampf handelt, bei dem man zusieht, wie Punkte gemacht werden. Ist es nicht auch so, dass es doch interessant ist zu beobachten, wenn mit der unterschiedlichen Wahrnehmung literarischer Texte unterschiedliche Weltsichten aufeinandertreffen? 
I.M.: Beides kommt zusammen. Dann also doch noch einmal phänomenologisch, im Sinne von Kracauer: An einem solchen Sendeformat ist schön zu sehen, welche unterschiedlichen Weltbezüge zum Ausdruck kommen, wenn diese vier Menschen über Bücher reden. Aber will man wirklich etwas über die Welt erfahren, wenn man Karasek lauscht? Das müsste man dann sehr fein analysieren, das kann man mündlich gar nicht beschreiben, was man da erfahren will. Er ist ein Typus, den der Zuschauer abgespeichert hat: Er steht für eine gewisse Lebensfreude, Harmonie, Kalaueranfälligkeit. Das Vergnügen beim Betrachten der Sendung besteht darin, dass dieses Bild der Typenkomödie, die sich der Zuschauer von den Beteiligten macht, immer wieder bestätigt wird. So wie man im Kasperle-Theater auch sehr genau weiß, was der Kaspar als Nächstes sagen wird. „Ach ja. Dem Karasek gefällt jetzt wieder, dass da eine hübsche junge Frau eine wichtige Rolle spielt, und das verpackt er in einen Kalauer“. ,Ja klar, die Löffler ist wieder ganz streng“. Es geht um die Bestätigung bestimmter Rollenbilder. Das Fernsehen hat logischerweise auch eine theatrale Dimension und man muss das Literarische Quartett auch so casten, dass unterschiedliche Typen vorkommen. Die erzählen dann verschiedene Weltzugänge. Wenn man es so beschreibt, ist es fast schon ein und dasselbe: der Battle-Charakter, wie ich vorhin gesagt habe, und das Weltbeschreibungsinteresse, das Sie benannt haben. Das fällt dann eigentlich zusammen.

S.B.: Stimmen Sie der Beobachtung zu, dass im Literarischen Quartett heute weniger konfrontativ gestritten wird?

I.M.: Die Zuspitzungskunst von Reich-Ranicki war gewaltig. Trotzdem hat man das Literarische Quartett auch deswegen so in Erinnerung, weil es zu dieser unschönen Szene zwischen Reich-Ranicki und Sigrid Löffler angesichts von Murakamis Roman Gefährliche Geliebte kam, bei der man heute immer noch fassungslos ist, wenn man sie sich wieder anschaut. So waren natürlich nicht alle Sendungen und Sigrid Löffler trat dann ja aus. Unsere Erinnerung ist selektiv und man erinnert besonders die dramatischen Momente. Aber es kann schon sein: das war seine große Begabung. Vor dem Literarischen Quartett hatte Reich-Ranicki den Bachmann-Wettbewerb in Klagenfurt geschaffen. Die Idee des Bachmann-Preises war auch eine Battle-Idee und die hat Marcel ReichRanicki entwickelt. Diese Rolle des pointierten Zuspitzers hatte er da schon erprobt - und vorher natürlich schon bei den Treffen der Gruppe 47. Das kam seinem Temperament und seiner Begabung - er hatte eine enorme rhetorische Begabung - sehr entgegen.

S.B.: Sie haben eine Vorlesung über das ,moralische Ich" in der Literaturkritik gehalten. Was hat es damit auf sich? 
I.M.: Das ist eine Gedankenfigur, die mich umtreibt. In Wahrheit sind alle Wortmeldungen von Menschen - Texte, was auch immer - von dem Bedürfnis getrieben, Recht zu behalten. Man kann sich ja fragen, warum sich die Leute überhaupt darüber streiten, ob ein Buch gut oder schlecht ist. Warum sie in diesem Urteil unbedingt Recht behalten wollen - es ist ja am Ende nur ein Buch. Ich denke, wir wollen Recht behalten, weil wir mit unseren Urteilen über Bücher gleichzeitig unseren eigenen Lebensentwurf verteidigen. Mir fiel das zum ersten Mal sehr deutlich auf, als ich noch ein junger Literaturredakteur war und feststellte, dass es die Neigung gab, dass die jüngeren Literaturkritiker die Urteile der älteren entwerteten, indem sie diesen vorhielten, ihr wisst halt nicht, was das „wahre Leben“ ist. Das Argument lief eigentlich doch hinaus auf: „Wir haben den besseren Sex“, und deswegen wissen wir es besser, können wir viel besser urteilen. Es gab zum Beispiel eine konfrontative Auseinandersetzung über den Debütroman von Helene Hegemann Axolotl Roadkill. Viele derer, die auf Hegemanns Seite standen und den Roman verteidigten, taten es, indem sie den Kritikern vorwarfen, ihr habt so ein langweiliges Leben, ihr könnt nur langweilige Bücher gut finden. Mit „,moralisch“ meinte ich also nicht, ,im moralisierenden Sinne“. Moral bedeutet immer die Verteidigung des eigenen Weltentwurfes. Der fließt natürlich zwingend in die Literaturkritik ein. Siehe Ein weites Feld bei Reich-Ranicki.

S.B.: Sigrid Löffler hat in einer Debatte über das zeitgenössische Feuilleton vor einer Entmachtung und Delegitimierung der Filmkritik und einem Statusverlust des Filmkritikers gesprochen, weil Hollywood die Filme mit enormem Werbeaufwand criticproof gemacht habe. Sie bedauert, dass der Konsument auf diese Weise zum Maßstab der Kritik gemacht werde und der ästhetische Gradmesser hingegen in den Hintergrund trete. Es gebe heute nicht nur keine Großkritiker mehr, was sie an sich nicht stört, sondern die Instanz des Kritikers habe maßgeblich an Bedeutung verloren.

I.M.: Darüber kann man stundenlang streiten. Ehrlich gesagt ist es mir gleichgültig, weil ich meine Arbeit als Literaturkritiker nicht darin sehe, darüber zu entscheiden, ob viele Menschen das Buch, das ich lobe, lesen, bzw. keiner das Buch in die Hand nimmt, das ich verrissen habe. Meine Literaturkritik versucht, einen Gedanken in die Welt zu bringen, einen Denkraum zu eröffnen. Welche Folgen das für den Kauf eines Buches hat, ist mir relativ egal. Diese präzeptorenhafte Rolle, das entspricht mir gar nicht.

S.B.: Kanonbildung, an der man als Literaturkritiker auch beteiligt ist, ist nichts, was Sie interessiert? 
I.M.: Kanonbildung ist ein sehr komplexer, ein unsteuerbarer Vorgang, an dem unendlich viele Parteien beteiligt sind. Und von den meisten wissen wir nicht, dass sie beteiligt sind. Deswegen können wir ihn nicht steuern. Ich bin immer hoch interessiert an Kanonisierungsprozessen und habe da keine Abwehrreflexe. Die Vorstellung hingegen, dass die Pflicht des Großkritikers darin bestünde, den Kanon festzusetzen, ist absurd. Kanonisierungsprozesse sind sozial strukturelle Vorgänge von größter Kontingenz.

S.B.: Sie waren Gastprofessor in St. Louis. Wie sehen Sie das Verhältnis von universitären und publizistischen Literaturkritikern?

I.M.: Sie kommunizieren wenig miteinander, was schade ist, denn das könnte fruchtbar sein. Es gibt an der FU in Berlin ein neues Exzellenzcluster Temporal Communities, das zu zeigen versucht, welche sozialen Verbindungen durch die Literatur geschaffen werden. Es gab zur festlichen Eröffnung eine Podiumsdiskussion, zu der ich eingeladen war. Da saßen Schriftsteller, Professoren, Literaturkritiker. Wir waren zu fünft. Das ist schon toll, weil alle eine andere Sprache und Beobachtungsformen haben, die sich gegenseitig befruchten. Das tut man aber ganz selten. Jeder ist sehr stark in seiner eigenen Blase.

S.B.: Marcel Reich-Ranicki hat oft Schriftsteller dazu animiert, für die FAZ zu schreiben, zum Beispiel Peter Rühmkorf. Machen Autoren andere Kritik als Profikritiker?

I.M.: Schon. Aber ich mag das. In Deutschland ist das ein bisschen zurückgegangen, weil sich die Schriftsteller ungerne dazu verdingen lassen. Es ist fast unmöglich, einen deutschen Schriftsteller dazu zu bringen, den Roman eines anderen Schriftstellers zu besprechen. Die Tradition kommt aus den USA. John Updike über den neuen Roman von Tom Wolfe. Norman Mailer über Gore Vidal. Die Amerikaner haben in vielerlei Hinsicht viel weniger Manschetten. Die Unterscheidung von Kritikern und Schriftstellern ist nicht so eng gefasst wie bei uns. Ich glaube, in Frankreich ist sie auch weniger eng gefasst. Reich-Ranicki hat das in seiner Zeit viel betrieben. Es ist ein bisschen schade, dass das in Deutschland zurückgegangen ist.

Bénédicte Terrisse: In Frankreich ist kürzlich ein Buch über Literaturkritik erschienen: Florian Pennanech, Poétique de la critique littéraire. De la critique comme littérature [Poetik der Literaturkritik. Von der Kritik als Literatur]. So wie damals Genette für die Erzählung die Erzähltheorie entwickelte, befasst sich Pennanech erstmalig mit der Literaturkritik als Genre und entwickelt eine Theorie dazu. Würden 
Sie auch sagen, dass Literaturkritik ein eigenes Genre und also auch Literatur ist?

I.M.: Man kann die Sache so oder so definieren, je nach Nomenklatur. Alles kann Teil der Literaturgeschichte sein, auch der politische Leitartikel. Tendenziell würde die avancierte Literatur das auch so sehen. Jeder Text ist Teil des textuellen Universums. Wenn Marcel Proust Contre SainteBeuve schreibt, lesen wir das heute natürlich auch als Text des Literaten Proust. Umgekehrt gibt es auch viel Literatur, die keine literarische Qualität hat. Gehört ein Roman, der keine literarische Qualität hat, zur Literaturgeschichte? Das könnte man auch umgekehrt fragen. Natürlich muss eine Rezension gut geschrieben sein. Kein Mensch liest irgendeinen Text, der nicht als Text Spaß macht, außer er wird unter Folter dazu gezwungen oder er muss es für die nächste Prüfung oder dergleichen lesen. Das ist jedoch nicht die Situation, die Sie am Samstagmorgen am Frühstückstisch haben, wenn die Zeitung auf Ihrem Tisch liegt. Im schlimmsten Fall lesen Sie eine schlecht geschriebene Rezension, weil Sie unbedingt wissen wollen: der neue Roman von Botho Strauß ist erschienen. Was sagt Die Welt dazu? Das Lesen der Rezension ist ihrerseits ein ästhetisches Vergnügen. Im Sinne dieser Frage gehört sie zur Literaturgeschichte. Die „Lust am Text", um mit Barthes zu sprechen, kann die Lust an einem Roman-Text wie an einer Rezension sein.

S.B.: In Frankreich hat jüngst ein Literaturwissenschaftler, Alexandre Gefen, die These aufgestellt, in der zeitgenössischen französischen Literatur sei ein Funktionswandel zu beobachten: nach dem Verlust der großen kollektiven Erzählungen habe sie eine, ,therapeutische“ Funktion übernommen. Er spricht davon, dass diese Literatur es ermögliche, das Besondere zu denken.

I.M.: Literatur, die eine kollektive therapeutische Funktion hat, mag es manchmal geben, aber man kann es nicht intendieren, planen. Bei der Vorstellung würde es mir grausen. Das Besondere zu denken hingegen ist nichts per se Therapeutisches. Er dreht ja die alte aristotelische Unterscheidung um. Bei Aristoteles ist die Geschichtswissenschaft für das Besondere zuständig und die Literatur für das Allgemeine. Man könnte sagen, die Literatur ist am Eigensinn interessiert, am NichtIdentischen im Adorno'schen Sinne. Sie ist stärker als der Journalismus in der Lage, einen anderen, neuen Ton zu bringen. Ja, Literatur als Medium des Besonderen in seiner Unberechenbarkeit und nicht Funktionalisierbarkeit, das macht sie aus.

B.T.: In Frankreich hatte man unter dem Einfluss von Roland Barthes die französische Literatur der 1980er Jahre als intransitiv aufgefasst, also ohne Gegenstand und Funktion. Gefen meinte, dass die heutige 
Gegenwartsliteratur sich davon abwende. Wie verhält es sich in der deutschen Gegenwartsliteratur?

I.M.: Deutschland stand in den 1980er - 1990er Jahren stark unter dem Einfluss französischer Theorie und das galt insbesondere auch für die deutsche Gegenwartsliteratur der damaligen Zeit. Für die Literatur der 1980er Jahre war das Intransitive ein ästhetisches Ideal, ab Mitte der 1990er Jahr sagte man: „Das hat die Literatur steril gemacht, keiner liest es mehr, Wirklichkeitsverlust, die Romane erzählen nichts mehr von der Welt, in der wir leben“. Ende der 1990er kam die Gegenbewegung mit dem Stichwort „die neue Lust am Erzählen“ und dem Kollektivbegriff „Fräuleinwunder“ auf, weil damals viele junge Frauen anfingen zu schreiben - und zwar sehr nah an ihrer Lebenswelt. Sie schrieben weniger hermetisch, nicht akademisch verkopft, aus ihrer Lebenswelt heraus. Seither ist die Literatur extrem in die andere Richtung gegangen. Das intransitive Sprachkunstwerk schafft es kaum mehr, Aufmerksamkeit zu erzielen. Das ist eigentlich ein Trauerspiel.

S.B.: Reich-Ranicki schreibt in Nicht nur in eigener Sache, die Deutschen hätten ein neurotisches Verhältnis zur Kritik und führt dies auf die verspätete Demokratie in Deutschland zurück.

I.M.: Ich glaube nicht, dass Deutschland im Hinblick auf die Kritik eine verspätete Nation ist. Ich weiß nicht, ob Frankreich jemanden wie Lessing hatte, der die Kritik so stark machte. Das würde mich wundern. In der Frühromantik gab es Schlegel, dann Fontane. Eigentlich stehen wir nicht schlecht da.

S.B.: Eine letzte Frage: In Frankreich gibt es eine Reihe von Autoren, die sich dem Medienkontakt vollkommen verweigert haben...?

I.M.: Für die Literatur finde ich das oft fruchtbar. Wir haben den Fall in Deutschland mit Botho Strauß. Ganz viel der Kraft und Originalität von Botho Strauß kommt auch aus seiner radikalen Medienabstinenz.

S.B. / B.T.: Herr Mangold, wir bedanken uns herzlich für das Gespräch.

I.M.: Sehr gern.

Das Gespräch fand am 29. Oktober 2019 in Berlin statt. 